\title{
INFRAESTRUCTURA HIDRÁULICA Y CRECIMIENTO ECONÓMICO EN LA MARINA BAJA (ALICANTE)
}

\author{
Cipriano JUÁREZ SÁNCHEZ-RUBIO \\ Universidad de Alicante
}

\section{Un espacio contrastado de recursos hídricos}

No existe correspondencia exacta entre las divisiones comarcales realizadas por geógrafos y las propuestas formuladas por economistas, lingüistas $\mathbf{u}$ organismos relacionados con la producción agraria. La administración agraria parcelaba recientemente el solar alicantino en diez unidades territoriales. La comarca geográfica de la Marina Baja se fragmentaba en dos áreas sumamente contrastadas según el valor de los índices agrícola, ganadero, regadío, barbecho y producto bruto por hectárea labrada ${ }^{1}$. Las dos zonas agrarias tenían como centros urbanos principales a Callosa de Ensarriá (montaña) y Villajoyosa (costa).

La montaña $(A l-\hat{Y} a b a l)$ y la costa (Al-Sahil) son los protagonistas geográficos de La Marina Baja ${ }^{2}$. Seguramente en estos dos Iqlim o climas agrícolas, según la división árabe, se fraccionaba el territorio. En el sector montañoso y de mayor altitud la precipitación anual varía entre 500 y 1.000 milímetros. Durante el año hidrológico existe un período (noviembre-marzo) de exceso de agua que se regula natural o artificialmente para atender a las demandas generadas por la actividad económica y el suministro urbano. La confluencia en el sector costero de la

${ }^{1}$ Juárez Sánchez-Rubio, C., "Utilización agraria del suelo en las comarcas de Alicante", Investigaciones Geográficas, $\mathrm{n}^{\circ} 1$ (1983), pp. 67-85.

${ }^{2}$ Rubiera, $\mathrm{M}^{2}$ J. y Epalza, M., Los nombres árabes de Benidorm y su comarca, Alicante, Universidad de Alicante, 1982, pág. 27 y siguientes. 
menor precipitación anual, de 300 a 370 milímetros, y la mayor evaporación, de 900 a 1.000 milímetros, explica el déficit hídrico estructural que soporta el marco de mejores condiciones térmicas. Tales diferencias internas determinan la necesidad de un aprovechamiento conjunto e integral de los recursos hidráulicos materializados en el cambio del modelo económico territorial. El resultado se traduce en el aumento demográfico, cambio en la distribución sectorial de la población activa, crecimiento de la tasa de empleo y mejora del nivel económico.

Los caracteres climáticos y los materiales geológicos determinan las especiales condiciones hidrológicas de sus cauces fluviales más significativos: Guadalest (río de piedras llanas) y Algar (cueva donde nace el río). La naturaleza no ha sido pródiga en llanuras (ausencia de nombres árabes) con el territorio, pero sí en topónimos referentes al relieve y al agua. El primero en sus acepciones de montañas, peñas, laderas, hoyas, etc. El segundo en la doble vertiente del recurso: en estado libre (río, torrente, arroyo, manantial) o en estado dominado (pozo, fuentes), además de la acción humana (arcos, puente) para conducir o trasvasar agua dentro de la comarca. Un conocimiento de inestimable valor geográfico aportado en la obra de los profesores Rubiera y Epalza citada.

Los recursos teóricos renovables, superficiales y subterráneos, sobre una reserva útil de 50 milímetros se estima en $50 \mathrm{Hm}^{3}$. Las aguas superficiales aportan el $60 \%$ y las subterráneas el $40 \%$ restante, pero su régimen natural de explotación sólo permite un aprovechamiento del $40 \%$ de la escorrentía total.

La mejor valoración de las condiciones naturales por los habitantes y la forma de superar sus limitaciones productivas agrarias se materializan en la sustitución de una economía agraria tradicional asentada en el policultivo de secano por una comercializada. Este cambio se traduce en aumentar los bajos indices de caudal regulado. Por tal motivo se procede a la movilización del recurso sobre la base de la orientación claramente productivista que evoluciona hacia la actividad de los servicios, principalmente el turismo, en expansión desde los años cincuenta. 


\section{Infraestructura hidráulica, competencia de usos y nuevo modelo económico.}

El crecimiento de la población, los cambios operados en los niveles de consumo y la ampliación de la superficie de riego impulsan la creación de una infraestructura hidráulica que resuelva el problema de la escasez y sea rentable económicamente. Los pantanos de Amadorio y Guadalest en funcionamiento desde 1957 y 1965, respectivamente, estaban contemplados en el Plan General de Riegos de la provincia de Alicante de 1940. Éstos tenían como objetivo fundamental regular las aguas superficiales para garantizar la dotación necesaria del espacio a transformar en regadfo, cuya cuantía varía de algo más de 5.000 hectáreas en 1960 a unas 7.600 hectáreas en 1990.

El aumento demográfico juega un papel decisivo en la expansión urbana-turística. La población censada varía de casi 48.000 habitantes en 1950 a 105.000 en 1991. Pero la población total registrada en la comarca durante el período de diciembre a abril es de 170.000 personas que se elevan a 190.000 en los meses de mayo, junio, octubre y noviembre para alcanzar el máximo, de 350.000 a 400.000 , durante el período vacacional de julio, agosto y septiembre.

Como los recursos renovables son prácticamente constantes, ha sido necesario ampliar la infraestructura. La acción comporta mayor nivel de garantra que se traduce en un cambio de modelo económico territorial. El cambio se plasma en un aumento espectacular del recurso por el sector servicios. Por ello fue obligado modificar legislativamente la concesión pública del agua con prelación hacia el aprovechamiento urbano-nurístico en detrimento de la superficie a transformar en regadio.

Esta competencia por el uso del agua se inicia en la década de los sesenta cuando el espacio regado había conseguido su garantía de dotación por la puesta en funcionamiento de los pantanos de Amadorio y Guadalest. Las fuertes demandas urbano-turisticas de los municipios costeros, en especial de Benidorm, determinan la asignación, desde 1965, de una dotación de agua regulada para el suministro urbano. Estas disposiciones sobre el agua, justificadas por el desarrollo turístico y llevadas a cabo con caudales, en principio, cedidos a los regantes, han motivado frecuentes y continuas tensiones entre ambos colectivos, acentuadas en los períodos de sequía. 
La serie de acciones contempladas tenían como objetivo elevar el grado de garantra del suministro a los centros urbanos. El objetivo se ha conseguido, a pesar del aumento en el consumo durante los años ochenta y sin aportaciones exteriores al territorio. Pero este modelo de reparto del agua repercute en la inseguridad del recurso para el riego. La esperanza de los agricultores se cifra en el uso de las agua depuradas, entre 12 y $14 \mathrm{Hm}^{3}$, y la explotación de los caudales subterráneos hasta cubrir sus necesidades.

La infraestructura existente en los años setenta, a pesar del beneficio económico-social producido, no era suficiente para asegurar la demanda creciente. La sequía de la segunda mitad de esta década puso de manifiesto la debilidad del sistema hidráulico para garantizar las demandas urbanas y agrícolas. Esta limitación impulsa la creación de un organismo supramunicipal: El Consorcio de Aguas de la Marina Baja, responsable de la gestión futura del agua. El nuevo ente consigue la aprobación de un caudal de 1.500 litros/segundo de aguas sobrantes del río Algar para su almacenamiento en el pantano de Guadalest (Fig. $\mathrm{n}^{\circ}$ 1). El volumen concedido para uso de abastecimiento urbano se acrecienta con el aprovechamiento integral del río Algar. La realización de la obra permite almacenar, mediante la infraestructura del Canal Bajo del Algar, un volumen de agua en el pantano de Amadorio para atender a las demandas estacionales de Benidorm y Villajoyosa.

El Consorcio está integrado por ocho municipios de los 19 que pertenecen a la unidad territorial: Alfaz del PI, Altea, Benidorm, Callosa de Ensarriá, Finestrat (La Cala), La Nucía, Polop y Villajoyosa. El consumo anual ha variado de $14,74 \mathrm{Hm}^{3}$ en 1981 a $19,39 \mathrm{Hm}^{3}$ en 1993 con un máximo de $22,12 \mathrm{Hm}^{3}$ en 1988 en relación con el rebrote turístico de 1986 a 1989 y la crisis posterior. Un consumo de agua real consorciada que se eleva a $25 \mathrm{Hm}^{3} /$ año por las pérdidas originadas en la red de distribución. La mitad de los recursos teóricos renovables son consumidos por la población permanente y estacional de los ocho municipios mancomunados. El volumen asignado a cada centro urbano está en relación con los porcentajes de contribución a la realización de las infraestructuras: Benidorm 59.1\%, Villajoyosa $14.7 \%$, Altea $13.6 \%$ y los menores porcentajes a Polop $1 \%$ y Finestrat $0.8 \%$. 
Los ingresos brutos obtenidos por el turismo en los ocho municipios se estiman en 1994 en unos 240.000 millones de pesetas, el $8 \%$ de los ingresos turísticos totales del país en este año (Benidorm el 5.8\%), aunque la devaluación de la peseta reduzca las entradas reales a sólo el $80 \%$ del producto bruto. Esta cuantía económica se diferencia considerablemente de los 4.050 millones de pesetas obtenidos de la producción de las tierras cultivadas en 1991. Es la manifestación más clara de la desigualdad del valor añadido del agua en base a los usos. El metro cúbico aplicado en el sector agrícola produce 135 pesetas en 1991 mientras el mismo volumen utilizado en el suministro urbano e industria turística se eleva a 9.600 pesetas brutas, que reducidas al $80 \%$ se convierten en 7.660 pesetas. El metro cúbico de agua aprovechado por el sector servicios mejora la productividad entre 55 y 70 veces la correspondiente en el sector agrícola.

$\mathrm{La}$ agricultura pierde protagonismo ante el avance de la demanda de agua para el sector turístico, que no asume las limitaciones físicas de la comarca en lo que a producción de agua se refiere. El espacio turístico-residencial aprovecha las aguas limpias asignadas al riego mediante el cambio normativo y los convenios establecidos con los agricultores. La desventaja de la agricultura en competencia por el agua con la industria más próspera y menos contaminante puede conducir a la desaparición del sector agrícola como entidad económica. En la actualidad la superficie dominada por el riego se mantiene en base a la utilización de los caudales depurados y a la explotación de las aguas subterráneas de pequeño coste en la extracción.

El fenómeno turístico sigue sin asumir la problemática de la escasez al no tomar en consideración la necesidad de ahorro y la paralela optimización en un medio semiárido. La dinámica seguida por el medio rural es diferente al sustituir el sistema de riego tradicional (inundación o a manta) por el riego localizado en cualquiera de sus modalidades. Su mayor sentido de la economía del agua (reduce el consumo y ahorra costes) se contrapone al derroche de la población y los usos recreativos.

Los niveles de producción de agua en la comarca han superado el techo teórico (existe sobreexplotación de 10 a $12 \mathrm{Hm}^{3} / \mathrm{año}$ ) mientras la demanda sigue en expansión, no sólo por el crecimiento demográfico (inmigración) sino por las expectativas futuras en el sector turístico. Éste parece prometedor porque, al ser improbable el retorno al empleo 
tradicional, habrá de contarse con una creciente población protegida por los Estados en situación de ocio. Las expectativas son grandes y fundadas, pero la oferta de la Marina Baja está condicionada por la disponibilidad de agua.

En definitiva, la expansión del turismo y la paralela concentración urbana, así como los cambios operados en los niveles de consumo, han creado un nuevo modelo de desarrollo. Éste se conforma en la moderna manera de organizar y asignar los recursos hídricos con el objetivo de solucionar la problemática generada, para no frenar el desarrollo económico-social conseguido. Un modelo, al margen de los beneficios proporcionados, que puede provocar impactos en el medio natural, reducir el espacio de labrantío y aumentar la competencia entre el medio urbano y rural por la escasez del recurso agua.

El impacto producido en la comarca por el cambio de un modelo territorial tradicional asentado en la actividad agrícola y marinera por otro asentado en la expansión turística-residencial es significativo. La utilización de la variable económico-social como parámetro de diferenciación espacial se plasma en una nueva fragmentación de la unidad territorial. La tradicional división a base de los Iqlim o climas agrícolas, se sustituye por otra constituida por tres zonas desiguales: la cora litoral, la dasicora inmediata y la areocora mediata.

La primera zona, integrada por los municipios de Alfaz del PI, Altea, Benidorm y Villajoyosa, se individualiza como un territorio de privilegio en el general desajuste del crecimiento económico. En este espacio se concentra el mayor índice de desarrollo turístico-residencial, la agricultura más productiva y de exportación y la densidad superior a $400 \mathrm{~h} / \mathrm{Klm}^{2}$. Sin embargo en esta unidad territorial existe una neta disimetría entre, de un lado, el núcleo de Benidorm, mucho más dinámico, y el resto de la cora. La dasicora inmediata la conforman Callosa de Ensarriá, La Nucia, Guadalest y Polop, donde confluyen una agricultura de nivel productivo semejante al sector de la costa, menor nivel de desarrollo turístico-residencial y densidad entre 50 y 300 $\mathrm{h} / \mathrm{Klm}^{2}$. En la areocora mediata se localiza la agricultura menos productiva (condiciones físicas adversas), escasa implantación del modelo turístico-residencial y pequeña densidad, siempre inferior a los $50 \mathrm{~h} / \mathrm{Klm}^{2}$. 
En definitiva, las mejoras realizadas sobre la infraestructura tienen como objetivo fundamental el aprovechamiento integral de los escasos recursos hidrológicos. El aumento de la disponibilidad se traduce en un alza del nivel de garantía del suministro urbano-residencial con paralelo beneficio económico. La repercusión económica en el espacio es desigual dando lugar a la fragmentación territorial de las tres zonas mencionadas pero estrechamente relacionadas laboral y económicamente. $Y$ es que una considerable poblacion residente en los municipios de la dasicora y la areocora se desplazan diariamente a los centros urbanos de la zona costera para trabajar en el sector servicios o como obreros de la construcción, sin abandonar su lugar de residencia.

El nuevo modelo económico-social, de preferente localización marítima, irradia hacia el espacio interior a manera de fenómeno "spread" o difusor, creando dependencia laboral y económica con la costa. Por tal motivo la unidad territorial presenta una dinámica interna sumamente contrastada pero estrechamente relacionada y dependiente de la infraestructura hidráulica. Ésta garantiza el nivel económico conseguido e impulsa nuevas acciones para no frenar el desarrollo.

\section{La problemática de la satisfacción hídrica futura y su valoración económica.}

La dotación de agua al segmento turístico no es sólo una operación económica aconsejable desde la óptica nacional, sino de exigencia de la propia sociedad. La demanda turística tiene características específicas que la diferencian de la población permanente. Como el factor que más contribuye al desarrollo turístico está relacionado con los indicadores socioeconómicos, existe una gran dificultad en saber tanto la población visitante como los niveles de dotación por sus hábitos de consumo. No obstante, desde la correcta planificación de los recursos hidráulicos es necesario la determinación de las demandas urbanas estacional y permanente.

La política económica ha evolucionado de intervenciones aisladas a la idea de una visión conjunta para lograr los objetivos determinados. En el caso de los municipios turísticos-residenciales la finalidad principal es aumentar la disponibilidad de agua, elevar el grado de garantía actual y futura, escalonar su uso y obtener la mayor rentabili- 
dad sin alterar las condiciones medio-ambientales. Por tanto, se necesita actuar de tal forma que el agua no se convierta en el factor limitativo del desarrollo económico-social, ni en un grave problema sanitario y ecológico después de ser utilizada.

La búsqueda de alternativas necesarias para incrementar los caudales hídricos, su mejor utilización y la obtención del mayor beneficio económico son las estrategias más adecuadas en la forma de aprovechamiento del agua en las zonas de gran escasez. El cumplimiento de estas propuestas implica hacer grandes inversiones de capital, al estar justificadas en los aspectos técnicos, ecológicos (deterioro de uso con incidencia en el baño) y económicos (riqueza).

El aumento de la regulación no debe ser un fin en sí mismo como podría derivarse de una concepción reguladora uniforme, sino que es una creación destinada a ajustar la adecuación de la oferta a la demanda de agua para abastecimiento. $Y$ es que si no se soluciona el problema de la escasez mediante la toma de decisiones políticas, de nada sirve hablar de modernidad, plan de carreteras y otras acciones, si la ampliación de la demanda no puede ser atendida porque no hay oferta.

El consumo de agua en los municipios consorciados ha crecido un $50 \%$ de 1981 a 1988 . Se ha pasado de utilizar un volumen de casi 15 $\mathrm{Hm}^{3}$ a $22 \mathrm{Hm}^{3}$. Este incremento, el más alto de toda la provincia de Alicante, se ha cubierto sobre la base de los recursos propios de la comarca y la gestión realizada por el Consorcio. El espectacular crecimiento de la demanda no se ha producido de forma continuada en el tiempo, sino que el período cronológico se subdivide en dos etapas diferentes. La primera, caracterizada por la oscilación en el consumo, se extiende de 1981 a 1984. Durante este perfodo coinciden crisis económica y sequía con reducción del consumo en un $14 \%$, especialmente significativo en Benidorm con el $19.5 \%$.

La segunda etapa se caracteriza por el continuo aumento del consumo y abarca el trienio de 1986-1988. El despegue en la demanda se vincula con el rebrote turístico y la mayor presencia del recurso. A partir de 1989 se inicia una nueva etapa de disminución de la demanda que alcanza su punto de inflexión en 1993 con un cambio de dirección en 1994. Esta correlación positiva existente impulsa la búsqueda de soluciones. Como la demanda crece en desequilibrio con el territorio y no se quiere sustituir el modelo económico, se debe tender a la 
conformación de una política hidráulica que garantice el suministro actual y las previsiones futuras.

Las aguas superficiales aportan entre 30 y $35 \mathrm{Hm}^{3}$ mediante la infraestructura de los embalses de Amadorio, $5 \mathrm{Hm}^{3}$, Guadalest, $5 \mathrm{Hm}^{3}$, y la regulación forzada del róo Algar (impulsión a Guadalest y Canal Bajo) entre 20 y $25 \mathrm{Hm}^{3} / a$ ño. Como el volumen de agua facturada es un $25 \%$ superior por las pérdidas en la red, la cuantía total utilizada se aproxima a los $25,83 \mathrm{Hm}^{3}$ estimados por nosotros para 1995 y los $31,51 \mathrm{Hm}^{3}$ para el año 2.000 con carácter predictivo ${ }^{3}$.

La dinámica económica-social instaurada por el modelo territorial conformado sin perspectiva de cambio, aboga por aumentar la disponibilidad para no detener el desarrollo. Una toma de decisión en consonancia con el objetivo del Anteproyecto del Plan Hidrologico Nacional: pretender garantizar el crecimiento turístico del litoral mediterráneo. Una acción realizable mediante una serie de decisiones que afectan tanto al uso del recurso como a la reducción de pérdidas. También a las mejoras tecnológicas en la producción de agua mediante la previsión de un Plan Estratégico de Desalinización de las agua salobres y marinas ya que el coste de producción de un metro cúbico de agua potable es de 0.7 dólares y de un dólar, respectivamente ${ }^{4}$. Estas acciones contempladas no agotan la posibilidad de aumentar los caudales en el territorio, puesto que el Plan Hidrológico de la Cuenca del Júcar prevé la realización del trasvase de agua de aquel colector a la Marina Baja.

La primera propuesta se asienta en el aprovechamiento integral de los recursos del territorio con desplazamiento de uso del sector agrícola para atención urbana. Esta opción comporta, en una primera aproximación, la reducción del espacio productivo agricola, pese a la depuración de las aguas y posterior reutilización. No obstante, la incorporación de las diferentes mejoras técnicas derivadas de la reducción de pérdidas en la red de distribución y el cambio en el sistema de riego (Plan de la

${ }^{3}$ Juárez Sánchez-Rubio, C., "Situación y perspectiva del abastecimiento de agua en los municipios turísticos del litoral alicantino", en: Libro Blanco del Turismo en la Costa Blanca, Alicante, Cámara Oficial de Comercio, Industria y Navegación de Alicante, 1990, tomo II, pp. 101-118.

${ }^{4}$ Prats, D., "Desalinización de agua salobre y agua de mar. Una alternativa para obtener agua de calidad", Revista Canelobre, n²7, pág. 23 y siguientes. 
modernización de la agricultura de la Comunidad Valenciana) puede ampliar la superficie transformada.

De las propuestas de posible realización para disponer de mayor cuantfa hídrica destaca la del trasvase Júcar-Vinalopó-Marina. El Plan de Cuenca dice expresamente: "que una vez regulado el río con la puesta en marcha de las nuevas presas (Tous, Bellús y Escalona) y una vez modernizados los regadfos, los sobrantes serán destinados al Vinalopó". El volumen a transferir sería, en una primera fase, de 100

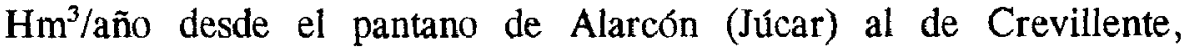
aprovechando la infraestructura del trasvase Tajo-Segura (Fig. $\mathbf{n}^{\circ} 2$ ). El objetivo es atender las demandas generadas en las comarcas alicantinas del Bajo y Medio Vinalopó, el Campo de Alicante y la Marina Baja.

El proyecto goza de larga tradición. El Concejo de Elche acuerda gestionar en 1420 la traída de aguas del Júcar, petición que se ha reiterado en sucesivas ocasiones, sin éxito alguno. En la actualidad el proyecto se contempla bajo el título de "corrección del déficit hídrico del Vinalopó". El caudal transferido será regulado en el pantano de Crevillente y bombeado a la comarca del Medio Vinalopó. Desde aquí se enviarán $15 \mathrm{Hm}^{3} /$ año al pantano de Amadorio (Marina Baja). El caudal a transferir tiene la finalidad de asegurar el suministro urbano de Benidorm y Villajoyosa, que arrojan un consumo asignado del $73.8 \%$ del agua del Consorcio. En la cristalización de esta obra se cifran las esperanzas de solucionar definitivamente el déficit hídrico estructural que soporta el territorio, en perfecta adecuación con los objetivos contemplados en el Anteproyecto del Plan Hidrológico Nacional.

El aumento de capacidad hidráulica proporcionado por las obras de defensa del Júcar mediante los embalses de Escalona, $87 \mathrm{Hm}^{3}$, Bellús $74 \mathrm{Hm}^{3}$, y la reconstruida presa de Tous, de 52 a $380 \mathrm{Hm}^{3}$, permitirá una regulación adicional que no era contemplada anteriormente ni está asignada a ninguna forma de utilización ${ }^{5}$. Además, esta mejora en el sistema hidráulico contribuirá a una mayor compatibilización de los aprovechamientos hidroeléctricos, instalados en su tramo medio, y el

${ }^{5}$ García, J. - Santafé, J. Ma - Cifres, E., "Efecto impulsor de las obras de defensa del río Júcar sobre el desarrollo agrícola regional", XIII Congreso de la Comisión Internacional de Riego y Drenajes, Casablanca, 1987 (Mecanografiado pág. 17). 
uso agrícola del tramo final, garantizando una explotación de agua que se estima técnicamente en $120 \mathrm{Hm}^{3} / a n ̃ o$ brutos con caudal continuo.

La mejor forma de optimizar económica y socialmente los nuevos recursos generados es su transferencia a las comarcas del Vinalopó en base a la compensación de la sobreexplotación de los acuíferos. En la Marina Baja como medio de atender el incremento no previsto en la demanda y auxilio en situaciones de sequía de los municipios turísticosresidenciales debido a su interés estratégico y económico. No obstante, en la realización del proyecto pesan una serie de inconvenientes derivados del escaso interés de ejecución temporal marcado por el Estado y el coste final de agua para los usuarios.

El primer inconveniente se plasma en la dilatación temporal y distribución de las remesas para su puesta en funcionamiento. De un coste de partida de 8.000 millones de pesetas se puede pasar a 10.000 millones, que en estrecha relación con las previstas inversiones, sólo 2.550 millones de pesetas hasta 1998 , hace que se alargue en el tiempo, año 1999, la finalización de la obra. Una dilación en el tiempo que obligará a la paralela explotación y competencia de uso de los escasos recursos de las comarcas para no frenar el desarrollo conseguido.

La segunda dificultad radica en el elevado precio final del agua a transferir. La necesidad de bombear los caudales desde los 130 m.s.n.m. en Crevillente (Bajo Vinalopó) hasta los 400 m.s.n.m. en el Medio Vinalopó y posterior envío al pantano de Amadorio (Marina Baja), junto con el canon de compensación a la empresa hidroeléctrica del Júcar, eleva a 37 ptas. el metro cúbico a utilizar en el conjunto Benidorm-Villajoyosa. Este elevado coste ha sido un poderoso incentivo para buscar otra alternativa que, arrancando del dispositivo hidroeléctrico Cortes-La Muela (Júcar), conduce el agua hasta los $400 \mathrm{~m}$. de altitud del Medio Vinalopó y posterior envío a la Marina Baja. El precio final sería de unas 31 ptas. por metro cúbico. $Y$ ambas alternativas de corrección del déficit hídrico pueden ser sustituidas por otra que contempla la transferencia de los caudales desde la reconstruida presa de Tous (Valencia) y a través de las Marinas: Alta (Denia) y Baja (Benidorm) hasta el Vinalopó (Villena-Elda-Elche). Una toma de decisión a la que nunca deberá añadirse las de orden legal provenientes de las concesiones otorgadas a los regantes del Júcar y sus derechos históricos, si es que llegaran a plantearse. 
En definitiva, la necesidad de solucionar la satisfacción de la demanda actual y futura de agua en cantidad y calidad en la Marina Baja pasa por: 1) La mejor gestión de los recursos disponibles mediante empresas supramunicipales (Consorcio) que garanticen el servicio y aprovechamiento integral. 2) La búsqueda de alternativas para incrementar la disponibilidad mediante la aplicación de las diferentes técnicas. 3) La realización del trasvase Júcar-Vinalopó por mejora económico-social y cumplimiento del principio de solidaridad.

\section{CONCLUSIONES}

1.- El cambio de modelo territorial producido en la comarca desde los años cincuenta está promovido por la mayor utilización del recurso agua hacia el sector servicios, especialmente turístico, en expansión.

2.- La tradicional división, a base de los dos Iqlim o climas agrícolas, se sustituye por otra constituida por tres zonas desiguales económica y socialmente: cora litoral, dasicora inmediata y aerocora mediata. Las tres están estrechamente relacionadas laboral y económicamente.

3.- La potenciación del modelo urbano-turístico-residencial está limitada por la disponibilidad en la garantía de agua. La superación de este condicionamiento se asienta en la mejor gestión de los recursos disponibles, la incorporación de nuevas tecnologías y la cristalización del trasvase Júcar-Vinalopó en cualquiera de sus alternativas.

4.- La utilización de trabajos de investigación y medios de comunicación social para explicar la situación a la que conduce el ritmo desaforado de consumo de agua en un medio semiárido. 


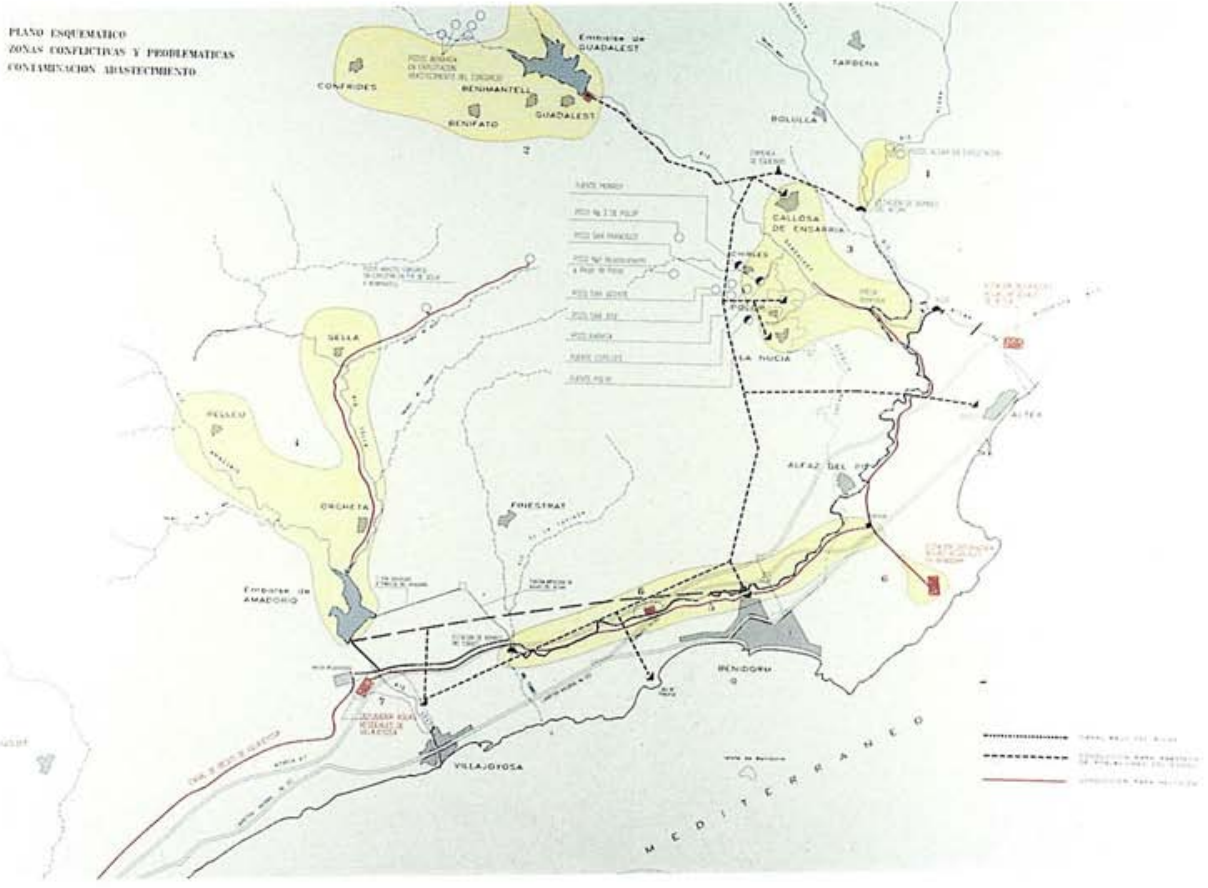

Fig. 1

Infraestructura hidráulica en la Marina Baja. 


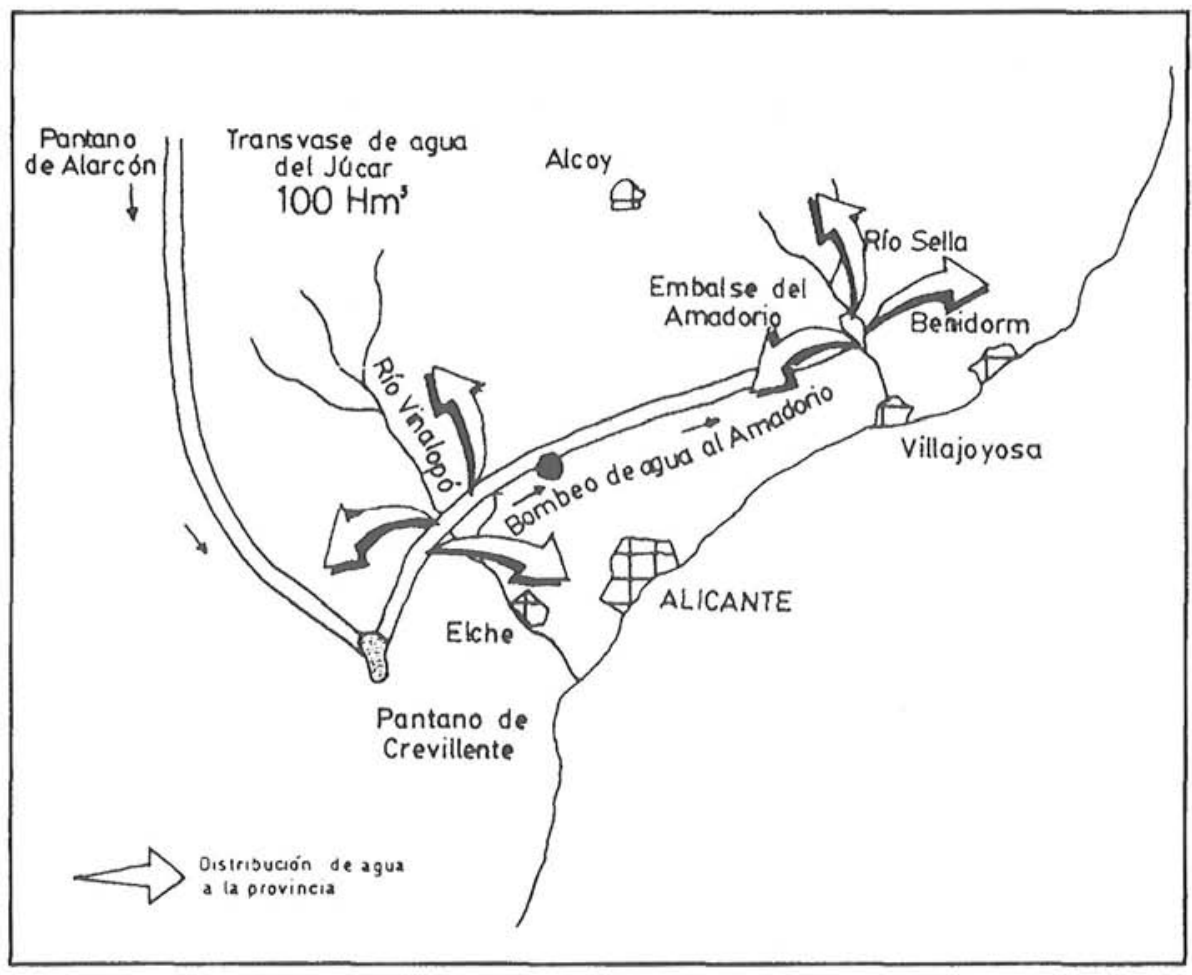

Figura $n^{\circ}$ 2.- Proyecto trasvase Júcar-Vinalopó-Marinas 